REVIEW

\title{
Pathobiology of brain metastases
}

\author{
N Nathoo, A Chahlavi, G H Barnett, S A Toms
}

J Clin Pathol 2005;58:237-242. doi: 10.1136/icp.2003.013623

Brain metastasis is a major cause of systemic cancer morbidity and mortality. Many factors participate in the development and maintenance of brain metastases. The survival of the metastasis depends upon crucial interactions between tumour cells and the brain microenvironment during its development at the new site. This review focuses on the pathobiological mechanisms involved in the establishment and regulation of brain metastases. Developments in molecular biology have vastly expanded our knowledge about the mechanisms of invasion, proliferation, metastatic cell signalling, and angiogenesis in brain metastases. Advances in this understanding of the pathobiology of brain metastasis may lead to novel targeted treatment paradigms and a better prognosis for patients with brain metastatic disease.

See end of article for authors' affiliations

.....................

Correspondence to: Dr S A Toms, Section of Metastatic Disease, Brain Tumor Institute, Cleveland Clinic Cancer Center R20 44195, Cleveland, Ohio, USA; tomss@cc.ccf.org

Accepted for publication 22 September 2004
M etastasis is a major cause of morbidity and mortality in human malignancies. ${ }^{1}$ The incidence of metastases to the brain is estimated to be about 170 000/year in the USA, an incidence 10 times higher than that of reported that between $20 \%$ and $40 \%$ of patients with systemic cancer will develop metastases involving the central nervous system (CNS) during the course of their disease. ${ }^{2}$ In adults, metastases to the brain most commonly arise from primary tumours of the lung (50-60\%), breast (15-20\%), skin (melanoma) (5-10\%), and gastrointestinal tract $(4-6 \%) .{ }^{3}{ }^{4} \mathrm{New}$ therapeutic strategies for systemic cancer and improvements in neuroimaging have resulted in increased detection rates for brain metastases. ${ }^{2}$

"The incidence of metastases to the brain is estimated to be about 170 000/year in the USA, an incidence 10 times higher than that of primary malignant brain tumours"

Current systemic treatments have mostly failed to treat brain metastases effectively. The blood-brain barrier (BBB) remains a formidable obstacle to the entry of most chemotherapeutic agents into the brain parenchyma. Incremental improvements in survival have been achieved for those patients with a limited number of lesions. These improvements have almost exclusively relied upon advances in local treatment for those patients with oligometastatic brain disease, such as image guided surgery and stereotactic radiosurgery. Unfortunately, individuals with multiple metastatic lesions and/or leptomeningeal disease primary malignant brain tumours. ${ }^{2}$ It has been usually succumb rapidly to brain metastasis, with life expectancy currently limited to several weeks to months. Despite the crucial importance of brain metastasis in cancer morbidity and mortality, much remains to be learned about the pathobiology of brain metastases. This review will focus on the current knowledge of the principal molecular and genetic processes responsible for the pathobiology of brain metastases.

\section{THE BRAIN METASTATIC PROCESS}

Metastasis of cancer cells is a highly selective, non-random process consisting of a series of linked sequential events. Various molecular and genetic changes define the multistep tumour dissemination process, which has been described as the "metastatic cascade". ${ }^{5}$ The steps involved in the cascade include escape from the primary tumour by invasion of the surrounding tissue, entry into and survival in the bloodstream (intravasation), arrest and/or extravasation at the secondary site and, finally, survival and proliferation to overt clinical metastases. ${ }^{6-8}$ For a cell to form successful haematogenous metastases to the brain, it must complete every step of the cascade.

Haematogenous metastasis is an inherently inefficient process. $^{59}$ Successful metastasis is dependent upon the interaction of tumour cells with the host defences and the brain microenvironment. Many characteristics of primary tumours predispose to haematogenous dissemination. An immature neovascular system, ${ }^{10}$ high interstitial pressure, ${ }^{11}$ and the close proximity of cancer cells to blood vessels ${ }^{12}{ }^{13}$ are factors favouring tumour cell intravasation. Once in the circulation, the intravascular tumour cells are subject to non-specific mechanical forces, such as haemodynamic turbulence, which might cause mechanical destruction of the cells, either before or during the extravasation process.

Primary tumours can be regarded as a genetically heterogeneous group of cells containing subpopulations of cells with varying degrees of metastatic potential, such as their invasive, angiogenic, and adhesive capacity, and CNS affinity. The brain is a unique target organ

Abbreviations: $\mathrm{BBB}$, blood-brain barrier; CNS, central nervous system; ECM, extracellular matrix; $\mathrm{HS}$, heparan sulfate; LOH, loss of heterozygosity; MMP, matrix metalloprotease; MSG, metastasis suppressor gene; NSCLC, non-small cell lung cancer; NT, neurotrophin; PAI-1/2, plasminogen activator inhibitor type 1/2; TIMP, tissue inhibitor of metalloproteinase; tPA, tissue-type plasminogen activator; UPA, urokinase-type plasminogen activator; uPA-R, uPA, urokinase-type plasminogen activator receptor; VEGF, vascular endothelial growth factor 
because it is surrounded by the BBB and lacks lymphatic drainage. The microenvironment of the brain differs from that of lung, liver, or bone. It is bathed in an interstitial fluid that is high in chloride, an environment that may not be conducive to many potential metastatic clones from cells of epithelial origin. It may be that the unique milieu of the brain attracts cells of neuroepithelial origin, such as small cell carcinoma of the lung or melanoma. It has been suggested that the common involvement of the CNS in metastatic melanoma may be the result of a "homing" influence, because melanocytes and neuronal subpopulations share a common embryological origin. ${ }^{14}$ To produce brain metastases, tumour cells must reach the brain vasculature by attaching to the microvessel endothelial cells, extravasate into the brain parenchyma, induce angiogenesis, and proliferate in response to growth factors. ${ }^{15} 16$

\section{Peritumorous brain events}

The borders between a brain metastasis and the surrounding brain parenchyma are usually distinct. Unlike gliomas, which diffusely infiltrate the brain parenchyma, metastatic tumours are usually sharply demarcated from the surrounding neuropil. Nonetheless, the tumour cells react with the surrounding brain parenchyma and blood vessels, resulting in a series of pathological and biochemical changes.

\section{Local tumour invasion}

Local brain invasion is a multifaceted process of tightly controlled mechanisms including cell motility, adhesion, and enzymatic remodelling of the extracellular matrix (ECM) components. Brain invasion requires paracrine interactions between brain stromal and endothelial cells and the invading metastatic tumour cells. Proteolytic degradation of the ECM is thought to aid tumour invasion by clearing a pathway for the invading tumour cells. The proteolytic activity is concentrated in the region of the cell membrane on the advancing edge of the invading tumour cell. ${ }^{17}{ }^{18}$ In addition, the proteolytic activity probably releases several factors from the ECM that promote cell proliferation and angiogenesis. Once in the brain parenchyma, the metastatic cell must adhere to the substratum to survive.

The E-cadherin-catenin complex is a prime mediator of cell-cell adhesion and is crucial for intercellular adhesiveness, and the maintenance of normal and malignant tissue architecture. Reduced expression of this complex has been associated with tumour invasion, metastasis, and an unfavourable prognosis. ${ }^{19}$ Recently, a significant correlation between E-cadherin expression and high MIB-1 index was reported for metastatic adenocarcinomas showing consistent E-cadherin expression, regardless of the degree of differentiation or the extent of spread of the disease. ${ }^{20}$ Integrins are major adhesion and signalling receptor proteins that mediate cell migration and invasion. They also trigger a variety of signal transduction pathways and regulate cytoskeletal organisation, specific gene expression, growth control, and apoptosis (programmed cell death). The blocking of integrin $\alpha 3 \beta 1$ in an animal model using a human non-small cell lung cancer (NSCLC) cell line significantly decreased brain metastasis. $^{21}$

Neurotrophins (NTs) are important because they stimulate brain invasion. In brain metastatic melanoma cells, NTs can promote invasion by enhancing the production of ECM degradative enzymes, such as heparanase, an enzyme capable of locally destroying both the ECM and the basement membrane of the BBB. Heparanase is an endo- $\beta$-d-glucuronidase that cleaves the heparan sulfate (HS) chains of ECM proteoglycans, and it is a unique metastatic determinant because it is the dominant mammalian HS degradative enzyme. ${ }^{22}$ Furthermore, it has been reported that p75 neurotrophin receptor, a common receptor for members of the NT family, is a molecular determinant of brain metastasis. ${ }^{23}$ Increased NT concentrations have also been reported at the tumour-brain interface of human brain melanoma. ${ }^{24}$

\section{Plasminogen activators and their inhibitors}

Since the initial report by Astedt et al in $1976^{25}$ that urokinase-type plasminogen activator (uPA) is produced and released from cancer cells, strong clinical and experimental evidence has accumulated that the tumour associated serine protease plasmin, its activator $\mathrm{UPA}$, the receptor UPA-R (CD87), and the inhibitors plasminogen activator inhibitor type 1 and 2 (PAI-1/2) are linked to cancer invasion and metastasis. Urokinase converts the zymogen plasminogen to plasmin, a trypsin-like enzyme with broad substrate specificities. UPA binds to the surface of the cell membrane and causes localised cell surface proteolytic activity, which is required for the destruction of the ECM, a vital step in tumour cell invasion. The proteolytic activity of UPA is modulated by its cell surface receptor and by PAI-1. Plasmin degrades components of the ECM such as fibrin, fibronectin, proteoglycans, and laminin, thereby allowing cells to migrate through the surrounding tissues. Plasmin also has the ability to activate other proteolytic enzymes, such as the metalloproteases.

"Plasmin degrades components of the ECM such as fibrin, fibronectin, proteoglycans, and laminin, thereby allowing cells to migrate through the surrounding tissues"

Bindal et al found high amounts of uPA in malignant glioma and metastatic tumours, whereas normal amounts were found in low grade gliomas. Neuroimaging analysis showed a significant correlation between high concentrations of UPA and necrosis and oedema. In addition, patients with high concentrations of UPA had shorter survival than those with low concentrations of uPA. Tissue-type plasminogen activator (tPA) was absent in colon, lung, and breast metastases, whereas metastatic melanoma had significantly more tPA activity than normal brain. They went on to conclude that high amounts of UPA and absent tPA activity correlated with malignant brain tumours, aggressive behaviour, and shorter patient survival. ${ }^{26}$ Localisation of PAI- 1 in proliferating vessels of brain metastases suggests that PAI- I may also be involved in angiogenesis. ${ }^{27} \mathrm{~A}$ variety of approaches to interfere with the expression or reactivity of UPA or UPA-R at the gene or protein level have been reported, including antisense oligonucleotides, antibodies, enzyme inhibitors, and recombinant or synthetic uPA and UPA-R analogues. ${ }^{28-30}$

\section{Matrix metalloproteases}

The matrix metalloproteases (MMPs) are a family of 20 enzymes that have the ability to degrade the ECM. Their activity results in the breakdown of connective tissue barriers such as collagens, laminins, fibronectin, vitronectin, and HS proteoglycans. Most MMPs are made by stromal cells (inflammatory cells, endothelial cells, and fibroblasts), although their expression is regulated by tumour cells via cytokines and the ECM metalloprotease inducer. Extracellular MMP inducer is found on the surface of tumour cells and stimulates the production of MMPs, thereby leading to ECM degradation and tumour cell motility. MMP activity has been found to correlate with invasiveness, metastasis, and poor prognosis in a variety of metastatic tumours. ${ }^{31}$

Jaalinoja et al, in their series of gliomas and metastases, reported that all metastatic brain tumours were positive for MMP-2. A correlation between MMP-2 expression and survival was found in malignant brain tumours. The mean survival of patients with MMP-2 negative tumours was 36 
months, and 7-14 months for patients with MMP-2 positive tumours. ${ }^{32}$ In another study, Arnold et al reported that MMP9 was upregulated in all brain metastases and primary brain tumours. However, no correlation with survival was possible. ${ }^{33}$ In mice overexpressing tissue inhibitor of metalloproteinase 1 (TIMP-1), brain metastases were significantly reduced by $75 \%$ compared with their wild-type littermates, demonstrating that ectopic TIMP-1 expression exerts a suppressive effect on metastasising tumour cells. ${ }^{34}$

\section{Neoangiogenesis and metastasis}

Angiogenesis is necessary for continued tumour growth. Failure of vascular growth will restrict the tumour mass to a size mediated by the limits of tissue diffusion distance, namely: $0.2 \mathrm{~mm} .^{35}$ The onset of angiogenesis within small clusters of tumour cells, known as the "angiogenic switch", is influenced by a complex interplay of proangiogenic molecules - such as vascular endothelial growth factor (VEGF) and the angiopoietin family-and antiangiogenic molecules, such as angiostatin. MMPs and TIMP also appear to be regulators of the angiogenic process, both by releasing proangiogenic peptides from the ECM and degrading the ECM to allow for the ingrowth of new blood vessels. Recently, the switch from vascular quiescence to angiogenesis has been shown to involve MMP-9/gelatinase B, which was found to be upregulated in angiogenic islets, with the tumours releasing VEGF-A from an extracellular reservoir. ${ }^{36}$

Further evidence for the role of VEGF in brain metastases has been reported by Kim et al, who in an animal model concluded that raised VEGF expression contributes to the ability of breast cancer cells to form brain metastases. Targeting endothelial cells with a VEGF receptor specific tyrosine kinase inhibitor reduced angiogenesis and restricted the growth of the brain metastases. ${ }^{37}$ Yano et al, in an elegant nude mouse model using six different human cancer cell lines in conjunction with sense and antisense VEGF isoforms, reported that VEGF expression was necessary but not sufficient for the production of brain metastases. ${ }^{38}$

\section{Dormant brain metastases}

The fact that brain metastases from numerous primary tumours have been reported to occur months or years after their removal supports the concept of tumour dormancy. The dormant state is characterised by normal proliferation, increased apoptosis, and a paucity of angiogenesis. It has been suggested that these late occurring metastases may result from differences in the proliferative potential of a subgroup of cells in the growth arrested metastatic tumour, innate biological differences between the dormant metastatic cells, or could simply be a new manifestation of the metastatic cascade (metastases forming metastases). ${ }^{39}$ Furthermore, it has been proposed that the angiogenic potential, in addition to the balance between proliferation and apoptosis in dormant brain metastases, may be influenced by antiangiogenic mediators released from the primary site, with primary tumour removal resulting in the loss of this antiangiogenic influence (endogenous inhibition), and sudden growth of the brain metastases as a result of an upregulation in angiogenesis. ${ }^{39}$

\section{Molecular regulation of metastatic growth}

The brain and its supporting structures represent a unique environment for metastatic growth. The unique properties of the CNS may explain the propensity of selected tumours, such as melanoma, to metastasise to the brain. Molecular factors may be organ specific and influence the tumour cells with regard to gene and protein expression, growth dynamics, and responsiveness to treatment. Growth regulation of metastatic cells in secondary sites is a crucial aspect of their proliferation. Recently, increasing attention has been drawn to the metastasis suppressor genes (MSGs), which, although distinct from oncogenes, can spontaneously suppress metastatic growth at any point in the metastatic cascade.

Nm23 was one of the first MSGs to be described, and is found in breast cancer and melanoma. ${ }^{40}$ It encodes a nucleotide diphosphate protein kinase that appears to regulate cell growth by interacting with menin (a tumour suppressor gene product). ${ }^{41}$ This was the first identified member of a family of genes that now has eight members.

\section{"Patients with cutaneous melanomas with low expression of $\mathrm{nm} 23$ appear to be more at risk of developing brain metastases"}

In vitro studies recently showed that $\mathrm{Nm} 23$ overexpression results in reduced anchorage independent colonisation in response to transforming growth factor $\beta$, reduced invasion, and reduced motility in response to multiple factors and increased differentiation. Furthermore, it has been suggested that reduced signal transduction is involved in the metastasis suppression effects of $\mathrm{Nm} 23$, and that the histidine protein kinase activity of $\mathrm{Nm} 23$ is responsible. ${ }^{42}$ Patients with cutaneous melanomas with low expression of nm23 appear to be more at risk of developing brain metastases. ${ }^{43}$

KAIl (CD82), which localises to chromosome 1lp11.2, encodes a protein that belongs to the tetraspanin superfamily. KAll regulates adhesion, migration, growth, and differentiation and was noted to be downregulated during prostate cancer progression. ${ }^{44}$ Its expression is inversely correlated with breast metastases ${ }^{45}{ }^{46}$ and melanoma metastases. ${ }^{47}$ It has also been found to inhibit the crucial steps of invasion and motility in colon cancer cells. ${ }^{48}$ KAIl may suppress metastases by influencing signalling pathways, such as those driven by the epidermal growth factor receptor. The association between epidermal growth factor receptor and KAIl may influence the Rho GTPase pathway, ${ }^{49}$ and result in the suppression of lamellipodia and migration signalling. ${ }^{50}$

CD44, which localises to chromosome 11 pl1.2, encodes an integral membrane glycoprotein that acts as a receptor for hyaluronic acid and osteopontin and influences cell adhesion. ${ }^{51}$ It has been suggested that CD44 acts by regulating the adhesion of circulating cancer cells to the endothelium at the secondary site, with the help of hyaluronate matrix ligand, or by its cytoplasmic attachment to actin associated proteins of the merlin/ezrin/radixin/moesin family. ${ }^{52}{ }^{53}$ DNA hypermethylation ${ }^{54}$ may be responsible for the downregulation of CD44, which is associated with higher tumour grade in clinical prostate cancer samples. ${ }^{55} \mathrm{CD} 44$ protein expression was reported to be upregulated in $48 \%$ of brain metastases studied. The most consistent expression of CD44 was seen in metastases from thyroid, skin, and breast cancer. ${ }^{56}$ Different CD44 expression patterns have been reported in primary brain tumours compared with brain metastases: exclusive expression of the standard form of CD44 was seen in primary brain tumours, whereas 22 of 26 brain metastases expressed the splicing variant of CD44. The authors concluded that the presence or absence of variant CD44 accounts for the high or low metastatic potential of human malignancies. ${ }^{57}$

MKK4, located on chromosome 14, is a mitogen activating protein kinase (MAPK) that is involved in signal transduction between MEKKl, protein kinase/JNKl, and p38 MAPK.$^{58}$ Previous studies have recorded that overexpression of the MKK4 gene results in the suppression of prostate cancer metastases, ${ }^{59}$ and an inverse relation between MKK4 staining and Gleason's score has been reported in prostate tumours. ${ }^{60}$ There is also evidence that the MKK4 gene acts as an MSG in ovarian cancer. ${ }^{61}$ 
Table 1 Summary of known metastastic tumour genes

\begin{tabular}{|c|c|c|c|}
\hline MSG & Chromosome location & Function & Metastatic tumour \\
\hline $\mathrm{Nm} 23$ & $17 q 21.3$ & Regulates cell growth, reduces invasion and motility & Breast and melanoma $^{31}$ \\
\hline KAIl (CD82) & $11 \mathrm{p} 11.2$ & $\begin{array}{l}\text { Regulates adhesion, migration, growth, and differentiation. } \\
\text { Influences signalling pathways such as EGFR and Rho } \\
\text { GTPases }\end{array}$ & Prostate, ${ }^{34}$ breast $^{35}{ }^{36}$ melanoma,${ }^{37}$ colon $^{38}$ \\
\hline CD44 & $11 \mathrm{p} 11.2$ & $\begin{array}{l}\text { Influences cell adhesion; hypermethylation of DNA results } \\
\text { in downregulation }\end{array}$ & Prostate, ${ }^{54}$ lung, breast, colon, melanoma ${ }^{56}$ \\
\hline MKK4 & 14 & $\begin{array}{l}\text { Regulates signal transduction between p38 and ERK/JNK } \\
\text { MAPKs }\end{array}$ & Prostate, ${ }^{5859}$ ovary $^{60}$ \\
\hline KISS-1 & 1 & $\begin{array}{l}\text { Inhibition of chemotaxis and invasion; G protein coupled } \\
\text { receptor ligand }\end{array}$ & Melanoma, breast ${ }^{6263}$ \\
\hline BrMS1 & 11 & Restores normal gap junction phenotype & Melanoma, ${ }^{64}$ bladder $^{66}$ \\
\hline RhoGD12 & $11 \mathrm{p} 11.2$ & Regulates RHO and RAC function & $\begin{array}{l}\text { Bladder, }{ }^{66} \text { prostate, bladder, breast, colorectum, } \\
\text { kidney, lung, liver }\end{array}$ \\
\hline PTEN/MMACl & $10 q 23$ & $\begin{array}{l}\text { Homologous to cytoskeletal protein tensin; involved in } \\
\text { tumour cell invasion and metastasis through interactions } \\
\text { of tensin with actin filaments at focal adhesions }\end{array}$ & $\begin{array}{l}\text { Breast, lung, colorectal, kidney, thyroid, } \\
\text { endometrial, germ cell, }{ }^{74} \text { melanoma }\end{array}$ \\
\hline
\end{tabular}

KISS- 1 is a gene that maps to chromosome 1 and which encodes metastin (fragment of KISS-1). Metastin, a ligand of the orphan G protein coupled receptor hOT7T175, inhibits chemotaxis, invasion, spreading, monolayer growth, and soft agar colony formation in transfected CHO cells. ${ }^{62}$ Enforced expression of KISS-1 suppressed melanoma and breast carcinoma metastases. ${ }^{63}$ Using in situ hybridisation, Shirasaki et al found an inverse correlation between KISS-1 expression and disease progression in clinical melanoma samples. ${ }^{64}$

BrMS1 is found on chromosome 11 and is expressed primarily in melanoma and breast cancer cells. It restores the normal gap junction phenotype, which aids in the maintenance of communication between cells within the primary tumour, thereby preventing disseminated tumour cell growth. ${ }^{65}$ In vitro studies have revealed that BRMS I expression inversely correlates with the metastatic potential of melanoma cells ${ }^{66}$ and reduces the growth of human bladder carcinoma cell lines. ${ }^{67}$

SSeCKS (pronounced essex), an acronym for Src-supressed $C$ kinase substrate, decreases the expression of VEGF through the reduction of AP-1, stimulates the expression of angiopoietin 1 , and may regulate BBB differentiation by modulating both brain angiogenesis and tight junction formation. SSeCKS is also significantly downregulated in human prostate epithelial cell lines. ${ }^{68}$

Drg-1 inhibits liver metastasis and invasion by colorectal carcinoma after DNA methylation inhibition, ${ }^{69}$ and an inverse correlation between Drg-l expression and Gleason's score has been reported in human prostate cancer specimens. ${ }^{70}$

RhoGDl2 (guanine nucleotide binding protein) has been designated an MSG. Increased RhoGD12 RNA expression in a bladder carcinoma cell line has been associated with decreased metastatic potential, ${ }^{67}$ and gene expression profiling of 105 clinical tumour specimens from multiple organ sites recorded an inverse correlation with the invasive potential of these tumours. ${ }^{71}$

\section{PTEN/MMAC 1}

This tumour suppressor gene has been designated PTEN (phosphatase and tensin homologue deleted on chromosome 10) or MMACl (mutated in multiple advanced cancers). Extensive homology exists between PTEN/MMACl and the cytoskeletal protein tensin, which is involved in tumour cell invasion and metastasis through its interactions with actin filaments at focal adhesions. ${ }^{72}$ Transfection experiments performed by Tamura et al recorded that overexpression of PTEN/ MMACl inhibited cell migration, whereas antisense PTEN/ MMACl enhanced migration. ${ }^{73}{ }^{74}$ Hahn et al performed loss of heterozygosity $(\mathrm{LOH})$ analysis and PTEN/MMACl sequence

\section{Take home messages}

- Many primary tumours metastasise to the brain, particularly those of the lung, breast, skin (melanoma), and gastrointestinal tract

- Metastasis of cancer cells occurs via the "metastatic cascade", namely: invasion of surrounding tissue, entry into and survival in the bloodstream (intravasation), arrest and/or extravasation at the secondary site, and survival and proliferation

- Developments in molecular biology have vastly expanded our knowledge about the mechanisms of invasion, proliferation, metastatic cell signalling, and angiogenesis in brain metastases, and several important molecules involved in these processes have been identified, such as E-cadherin, catenins, neurotrophins, plasminogen activators and inhibitors and matrix metalloproteases

- Recently, several metastasis suppressor genes (MSGs), which can spontaneously suppress metastatic growth at any point in the metastatic cascade, have been identified, such as nm23 and CD44

- These advances in the understanding of the pathobiology of brain metastasis may lead to novel targeted treatment paradigms and a better prognosis for patients with brain metastatic disease

analysis in 56 brain metastases from various primary tumours and found that despite an overall high rate of LOH $(50 \%)$, there was a low frequency of PTEN/MMACl mutation detection (14\%), indicating that one or more additional tumour suppressor genes may be present on chromosome $10 .{ }^{75}$ They also recorded a high proportion of inactivating mutations of PTEN/MMACl in lung cancer (25\%), suggesting that inactivation of the gene contributed to tumour progression in this subgroup of metastases.

In NSCLC, the incidence of LOH on $2 \mathrm{p}$ and $2 \mathrm{q}$ was found to be significantly higher in brain metastases than in primary tumours. ${ }^{76}$ The incidence of $\mathrm{LOH}$ on chromosome arms $2 \mathrm{q}$, 9p, 18q, and 22q in brain metastases was significantly higher than that seen in stage I primary lung tumours, and was thought to play an important role in the progression of NSCLC. ${ }^{77}$ Therefore, the inactivation of tumour suppressor genes on chromosome 2 appear to result in an aggressive phenotype of NSCLC cells. ${ }^{76} 77$ 
"Some metastasis suppressor genes (MSGs) are more likely to be turned off rather than mutated, suggesting that gene silencing via methylation or histone acetylation may be important in the regulation of MSGs"

Other MSGs whose functional importance has yet to be defined are: collapsin response mediator protein 1 , which is involved in cell movement; gelsolin, which modulates actin activity to regulate movement; maspin (mammary serine protease protease inhibitor); heterochromatin associated protein $1\left(\mathrm{HPl}^{\mathrm{HS} \alpha}\right)$; and SHP-2 (cytoplasmic tyrosine kinase phosphatase, involved in signal transduction)

Elimination of a single step in the metastatic cascade may halt the process of metastasis, so that restoration of MSG expression in competent tumour cells may produce clinical benefit in patients with brain metastases. It appears that some MSGs (NM23, MKK4, and KAI1) ${ }^{60} 7879$ are more likely to be "turned off" rather than mutated, suggesting that gene silencing via methylation or histone acetylation may be important in the regulation of MSGs. Therefore, strategies to restore gene expression by altering the proteins responsible or reintroducing genes or proteins not subject to this form of regulatory control may be necessary to restore suppressor function. ${ }^{80}$ Table 1 summarises the known metastatic tumour genes.

\section{CONCLUSION}

Insight into the molecular mechanisms regulating the pathobiology of brain metastases should improve our understanding of the complex interactions between metastatic tumour cells and the host environment. A new era of metastasis suppressor research is currently evolving. The identification of MSGs that have functional relevance in well defined animal models or studies with banked human tissues is necessary if we are to apply these experimental data in the clinical setting. Until strategies based upon the molecular biology of CNS metastasis are implemented, hope will remain slim for all but the rare patient with a controlled primary cancer and few CNS metastases.

\section{ACKNOWLEDGEMENTS}

The authors wish to thank M Tobin (CME and Editorial Services), Department of Neurosurgery, Cleveland Clinic Foundation for her assistance with the preparation of this manuscript.

\section{Authors' affiliations}

N Nathoo, A Chahlavi, G H Barnett, S A Toms, Brain Tumor Institute, Taussig Cancer Center and Department of Neurosurgery, Cleveland Clinic Foundation, 44122 Cleveland, Ohio, USA

\section{REFERENCES}

1 Fidler IJ, Ellis LM. The implications of angiogenesis for the biology and therapy of cancer metastasis. Cell 1994;79:185-8.

2 Landis SH, Murray T, Bolden S, et al. Cancer statistics, 1998. CA Cancer J Clin 1999;49:8-31.

3 Patchell RA. The management of brain metastases. Cancer Treat Rev 2003;29:533-40.

4 Wen PY, Loeffler JS. Brain metastases. Curr Treat Options Oncol 2000; 1:447-58

5 Fidler IJ. Critical factors in the biology of human cancer metastases: twentyeight G.H.A. Clowes memorial award lecture. Cancer Res 1990:50:6130-8.

6 Christiano AP, Yoshida BA, Dubauskas Z, et al. Development of markers of prostate cancer metastasis. Review and perspective. Urol Oncol 2000;5:217-23.

7 Poste G, Fidler IJ. The pathogenesis of cancer metastasis. Nature 1980;283:139-46.

8 MacDonald NJ, Steeg PS. Molecular basis of tumour metastasis. Cancer Surv 1993;16:175-99.

9 Chambers AF, MacDonald IC, Schmidt EE, et al. Clinical targets for antimetastasis therapy. Adv Cancer Res 2000:79:91-121.

10 Vaupel P, Kallinowski F, Okunieff P. Blood flow, oxygen and nutrient supply, and metabolic microenvironment of human tumors: a review. Cancer Res 1989;49:6449-65.
11 Boucher Y, Baxter LT, Jain RK. Interstitial pressure gradients in tissue-isolated and subcutaneous tumors: implications for therapy. Cancer Res 1990;50:4478-84

12 Xie K, Wang B, Shi Q, et al. Mouse models of metastatic pancreatic adenocarcinoma. Int J Pancreatol $2001 ; 29: 25-35$.

13 Shi Q, Xie K. Experimental animal models of pancreatic cancer [review]. Int J Oncol 2000; 17:217-25.

14 Marchetti D, Denkins Y, Reiland J, et al. Brain-metastatic melanoma: a neurotrophic perspective. Pathol Oncol Res 2003:9:147-58.

15 Nicolson GL, Menter DG, Herrmann JL, et al. Brain metastasis: role of trophic, autocrine, and paracrine factors in tumor invasion and colonization of the central nervous system. Curr Top Microbiol Immunol 1996;213(Pt 2):89-1 15.

16 Yano S, Shinohara H, Herbst RS, et al. Expression of vascular endothelial growth factor is necessary but not sufficient for production and growth of brain metastasis. Cancer Res 2000;60:4959-67.

17 Yamamoto M, Ueno Y, Hayashi S, et al. The role of proteolysis in tumor invasiveness in glioblastoma and metastatic brain tumors. Anticancer Res 2002;22:4265-8

18 Vassalli JD, Sappino AP, Belin D. The plasminogen activator/plasmin system. J Clin Invest 1991;88:1067-72.

19 Bremnes RM, Veve R, Hirsch FR, et al. The E-cadherin cell-cell adhesion complex and lung cancer invasion, metastasis, and prognosis. Lung Cancer 2002;36:115-24.

20 Shabani HK, Kitange G, Tsunoda K, et al. Immunohistochemical expression of E-cadherin in metastatic brain tumors. Brain Tumor Pathol 2003;20:7-12.

21 Yoshimasu T, Sakurai T, Oura S, et al. Increased expression of integrin alpha3betal in highly brain metastatic subclone of a human non-small cell lung cancer cell line. Cancer Sci 2004;95:142-8.

22 Marchetti D, Denkins Y, Reiland J, et al. Brain-metastatic melanoma: a neurotrophic perspective. Pathol Oncol Res 2003;9:147-58.

23 Marchetti D, Nicolson GL. Human heparanase: a molecular determinant of brain metastasis. Adv Enzyme Regul 2001;41:343-59.

24 Denkins Y, Reiland J, Roy M, et al. Brain metastases in melanoma: roles of neurotrophins. Neurooncology 2004;6:154-65.

25 Astedt B, Holmberg L. Immunological identity of urokinase and ovarian carcinoma plasminogen activator released in tissue culture. Nature 1976;261:595-7

26 Bindal AK, Hammoud M, Shi WM, et al. Prognostic significance of proteolytic enzymes in human brain tumors. J Neurooncol 1994;22:101-10.

27 Kono S, Rao JS, Bruner JM, et al. Immunohistochemical localization of plasminogen activator inhibitor type 1 in human brain tumors. J Neuropathol Exp Neurol 1994;53:256-62.

28 Reuning U, Sperl S, Kopitz C, et al. Urokinase-type plasminogen activator (UPA) and its receptor (UPAR): development of antagonists of UPA/UPAR interaction and their effects in vitro and in vivo. Curr Pharm Des 2003;9:1529-43

29 Sperl S, Mueller MM, Wilhelm OG, et al. The uPA/uPA receptor system as a target for tumor therapy. Drug News Perspect 2001;14:401-11.

30 Guthaus E, Schmiedeberg N, Burgle $M$, et al. The urokinase receptor (UPAR, CD87) as a target for tumor therapy: uPA-silica particles (SP-uPA) as a new tool for assessing synthetic peptides to interfere with uPA/uPA-receptor interaction. Recent Results Cancer Res 2003;162:3-14.

31 Starnenkovic I. Extracellular matrix remodelling: the role of matrix metalloproteinases. J Pathol 2003;200:448-64.

32 Jaalinoja J, Herva R, Korpela M, et al. Matrix metalloproteinase 2 (MMP-2) immunoreactive protein is associated with poor grade and survival in brain neoplasms. J Neurooncol 2000;46:81-90.

33 Arnold SM, Young AB, Munn RK, et al. Expression of p53, bcl-2, E-cadherin, matrix metalloproteinase-9, and tissue inhibitor of metalloproteinases-1 in paired primary tumors and brain metastasis. Clin Cancer Res 1999:5:4028-33.

34 Kruger A, Sanchez-Sweatman OH, Martin DC, et al. Host TIMP-1 overexpression confers resistance to experimental brain metastasis of a fibrosarcoma cell line. Oncogene 1998;16:2419-23.

35 Chang C, Werb Z. The many faces of metalloproteases: cell growth, invasion, angiogenesis and metastasis. Trends Cell Biol 2001;11:S37-43.

36 Bergers G, Brekken R, McMahon G, et al. Matrix metalloproteinase- 9 triggers the angiogenic switch during carcinogenesis. Nat Cell Biol 2000;2:737-44.

37 Kim LS, Huang S, Lu W, et al. Vascular endothelial growth factor expression promotes the growth of breast cancer brain metastases in nude mice. Clin Exp Metastasis 2004;21:107-18.

38 Yano S, Shinohara H, Herbst RS, et al. Expression of vascular endothelial growth factor is necessary but not sufficient for production and growth of brain metastasis. Cancer Res 2000;60:4959-67.

39 Kirsch M, Schackert G, Black PM. Angiogenesis, metastasis, and endogenous inhibition. J Neurooncol 2000;50:173-80.

40 Leone A, Flatow U, King CR, et al. Reduced tumor incidence, metastatic potential, and cytokine responsiveness of nm23-transfected melanoma cells. Cell 1991;65:25-35.

41 Yaguchi H, Ohkura N, Tsukada T, et al. Menin, the multiple endocrine neoplasia type 1 gene product, exhibits GTP-hydrolyzing activity in the presence of the tumor metastasis suppressor nm23. J Biol Chem 2002;277:38197-204

42 Ouatas $T$, Salerno M, Palmieri $D$, et al. Basic and translational advances in cancer metastasis: Nm23. J Bioenerg Biomembr 2003;35:73-9.

43 Sarris M, Scolyer RA, Konopka M, et al. Cytoplasmic expression of nm23 predicts the potential for cerebral metastasis in patients with primary cutaneous melanoma. Melanoma Res 2004;14:23-7.

44 Dong JT, Suzuki H, Pin SS, et al. Down-regulation of the KAll metastasis suppressor gene during the progression of human prostatic cancer 
infrequently involves gene mutation or allelic loss. Cancer Res 1996;56:4387-90.

45 Yang $X$, Welch DR, Phillips KK, et al. KAll, a putative marker for metastatic potential in human breast cancer. Cancer Lett 1997;119:149-55.

46 Phillips KK, White AE, Hicks DJ, et al. Correlation between reduction of metastasis in the MDA-MB-435 model system and increased expression of the Kai-1 protein. Mol Carcinog 1998;21:111-20.

47 Takaoka A, Hinoda Y, Sato S, et al. Reduced invasive and metastatic potentials of KAll-transfected melanoma cells. Jpn J Cancer Res 1998;89:397-404.

48 Takaoka A, Hinoda Y, Satoh S, et al. Suppression of invasive properties of colon cancer cells by a metastasis suppressor KAll gene. Oncogene 1998; 16:1443-53.

49 Shevde LA, Welch DR. Metastasis suppressor pathways - an evolving paradigm. Cancer Lett 2003;198:1-20.

50 Odintsova E, Sugiura T, Berditchevski F. Attenuation of EGF receptor signaling by a metastasis suppressor, the tetraspanin CD82/KAl-1. Curr Biol 2000;10:1009-12.

51 Braun S, Pantel K, Muller P, et al. Cytokeratin-positive cells in the bone marrow and survival of patients with stage I, II, or III breast cancer. N Engl J Med 2000;342:525-33.

52 Gao AC, Lou W, Sleeman JP, et al. Metastasis suppression by the standard CD44 isoform does not require the binding of prostate cancer cells to hyaluronate. Cancer Res 1998:58:2350-2.

53 Gao AC, Lou W, Dong JT, et al. CD44 is a metastasis suppressor gene for prostatic cancer located on human chromosome $11 \mathrm{p} 13$. Cancer Res 1997; 57:846-9.

54 Lou W, Krill D, Dhir R, et al. Methylation of the CD44 metastasis suppressor gene in human prostate cancer. Cancer Res 1999;59:2329-31.

55 Noordzii MA, van Steenbrugge GJ, Schroder FH, et al. Decreased expression of CD44 in metastatic prostate cancer. Int J Cancer 1999;84:478-83.

56 Harabin-Slowinska M, Slowinski J, Konecki J, et al. Expression of adhesion molecule CD44 in metastatic brain tumors. Folia Neuropathol 1998:36:179-84

57 Li H, Liu J, Hofmann M, et al. Differential CD44 expression patterns in primary brain tumours and brain metastases. Br J Cancer 1995;72:160-3.

58 Teng DH, Perry WL 3rd, Hogan JK, et al. Human mitogen-activated protein kinase kinase 4 as a candidate tumor suppressor. Cancer Res 1997; 57:4177-82.

59 Yoshida BA, Dubauskas Z, Chekmareva MA, et al. Mitogen-activated protein kinase kinase 4/stress-activated protein/Erk kinase 1 (MKK4/SEK1), a prostate cancer metastasis suppressor gene encoded by human chromosome 17. Cancer Res 1999:59:5483-7.

$60 \mathrm{Kim}$ HL, Vander Griend DJ, Yang X, et al. Mitogen-activated protein kinase kinase 4 metastasis suppressor gene expression is inversely related to histological pattern in advancing human prostatic cancers. Cancer Res 2001:61:2833-7.

61 Yamada SD, Hickson JA, Hrobowski Y, et al. Mitogen-activated protein kinase kinase 4 (MKK4) acts as a metastasis suppressor gene in human ovarian carcinoma. Cancer Res 2002;62:6717-23.

62 Hori A, Honda S, Asada M, et al. Metastin suppresses the motility and growth of $\mathrm{CHO}$ cells transfected with its receptor. Biochem Biophys Res Commun $2001 ; 286: 958-63$.
63 Lee JH, Welch DR. Suppression of metastasis in human breast carcinoma MDA-MB-435 cells after transfection with the metastasis suppressor gene, KiSS-1. Cancer Res 1997;57:2384-7.

64 Shirasaki F, Takata M, Hatta N, et al. Loss of expression of the metastasis suppressor gene KiSS1 during melanoma progression and its association with LOH of chromosome 6q16.3-q23. Cancer Res 2001;61:7422-5.

65 Seraj MJ, Samant RS, Verderame MF, et al. Functional evidence for a novel human breast carcinoma metastasis suppressor, BRMS1, encoded at chromosome 11q13. Cancer Res 2000;60:2764-9.

66 Shevde LA, Samant RS, Goldberg SF, et al. Suppression of human melanoma metastasis by the metastasis suppressor gene, BRMS1. Exp Cell Res 2002;273:229-39.

67 Seraj MJ, Harding MA, Gildea JJ, et al. The relationship of BRMS1 and RhoGDI2 gene expression to metastatic potential in lineage related human bladder cancer cell lines. Clin Exp Metastasis 2000;18:519-25.

68 Xia W, Unger P, Miller L, et al. The Src-suppressed C kinase substrate, $\mathrm{SSeCKS}$, is a potential metastasis inhibitor in prostate cancer. Cancer Res 2001:61:5644-51.

69 Guan RJ, Ford HL, Fu Y, et al. Drg-1 as a differentiation-related, putative metastatic suppressor gene in human colon cancer. Cancer Res 2000:60:749-55.

70 Bandyopadhyay S, Pai SK, Gross SC, et al. The Drg-1 gene suppresses tumor metastasis in prostate cancer. Cancer Res 2003;63:1731-6.

71 Gildea JJ, Seraj MJ, Oxford G, et al. RhoGDI2 is an invasion and metastasis suppressor gene in human cancer. Cancer Res 2002;62:6418-23.

72 Li J, Yen C, Liaw D, et al. PTEN, a putative protein tyrosine phosphatase gene mutated in human brain, breast, and prostate cancer. Science 1997;275:1943-7.

73 Tamura M, Gu J, Matsumoto K, et al. Inhibition of cell migration, spreading, and focal adhesions by tumor suppressor PTEN. Science 1998;280:1614-17.

74 Tamura M, Gu J, Takino T, et al. Tumor suppressor PTEN inhibition of cell invasion, migration, and growth: differential involvement of focal adhesion kinase and p130Cas. Cancer Res 1999;59:442-9.

75 Hahn M, Wieland I, Koufaki ON, et al. Genetic alterations of the tumor suppressor gene PTEN/MMAC1 in human brain metastases. Clin Cancer Res 1999;5:2431-7.

76 Otsuka T, Kohno T, Mori M, et al. Deletion mapping of chromosome 2 in human lung carcinoma. Genes Chromosomes Cancer 1996;16:113-19.

77 Shiseki M, Kohno T, Adachi J, et al. Comparative allelotype of early and advanced stage non-small cell lung carcinomas. Genes Chromosomes Cancer 1996;17:71-7.

78 Miyazaki T, Kato $H$, Shitara $Y$, et al. Mutation and expression of the metastasis suppressor gene KAll in esophageal squamous cell carcinoma. Cancer 2000;89:955-62.

79 Cropp CS, Lidereau R, Leone A, et al. NMEl protein expression and loss of heterozygosity mutations in primary human breast tumors. J Natl Cancer Inst 1994:86:1167-9.

80 Steeg PS. Metastasis suppressors alter the signal transduction of cancer cells. Nat Rev Cancer 2003;3:55-63.

81 Guldberg $\mathbf{P}$, thor Straten $\mathrm{P}$, Birck A, et al. Disruption of the MMACl/PTEN gene by deletion or mutation is a frequent event in malignant melanoma. Cancer Res 1997:57:3660-3. 